\title{
ATP Citrate Lyase and LncRNA NONMMUT010685 Play Crucial Role in Nonalcoholic Fatty Liver Disease Based on Analysis of Microarray Data
}

\author{
Tao-tao Ma ${ }^{a, b} \quad$ Cheng Huang ${ }^{a}$ Ying Nib You Yang ${ }^{b} \quad$ Jun Li $^{a}$ \\ ${ }^{a}$ Anhui Province Key Laboratory of Major Autoimmune Diseases, Anhui Institute of Innovative Drugs, \\ School of Pharmacy, Anhui Medical University, Hefei, bSchool of Clinical Medicine, Anhui Medical \\ University, Hefei, China
}

\section{Key Words}

NAFLD $\bullet$ LncRNAs • Gene ontology • Acly • Molecular mechanism

\begin{abstract}
Background/Aims: Nonalcoholic fatty liver disease (NAFLD) is the most common cause of liver disease with unclear molecular mechanisms. Our study intended to identify potential long non-coding RNAs (IncRNAs) and genes, and to determine the potential molecular mechanisms of NAFLD pathogenesis. Methods: The microarrays of GSE24031 and GSE57425 were downloaded from the Gene Expression Omnibus database. GSE24031 included 4 control and 4 model mice and GSE57425 included 3 control and 3 model mice on the basis of GPL1261 platform. Differentially expressed IncRNAs and mRNAs between control and NAFLD liver tissue were calculated. Gene ontology (GO), pathway enrichment analyses, co-expression network and PPI were performed to analyze the biological roles and pathways for the differentially expressed IncRNAs and mRNAs. Non-alcoholic steatohepatitis (NASH) rats were further chosen to investigate the key protein identified based on co-expression network and proteinprotein interaction (PPI) network data. Results: A total of 6 significantly up-regulated and 39 down-regulated IncRNAs, 340 up-regulated and 281 down-regulated mRNAs were identified. LncRNA-mRNA co-expression network were analyzed to show a total of 16 key IncRNAs (node degree $>10$ ) in NAFLD samples compared to control tissues. Three key protein identified on co-expression network and protein-protein interaction (PPI) network data were verified in NASH in vivo. The protein level of ATP-citrate lyase (Acly) was significantly increased while IncNONMMUT010685 and NONMMUT050689 in NAFLD samples, whose regulator gene was x-box binding protein 1 (XBP1) and receptor-interacting protein 1 kinase (RIPK1) respectively, were gradually reduced in NASH. Conclusion: In summary, we found a set of IncRNAs and mRNAs differentially expressed in the development of NAFLD. LncRNA Ttc39aos1 and Acly,
\end{abstract}

\footnotetext{
Jun Li Anhui Province Key Laboratory of Major Autoimmune Diseases, Anhui Institute of Innovative Drugs, School of Pharmacy, Anhui Medical University, No. 81 Meishan Road, Anhui (China); Tel. +86 55165161001, Fax +8655165161001, E-Mail lj@ahmu.edu.cn
} 


\section{Cellular Physiology Cell Physiol Biochem 2018;51:871-885

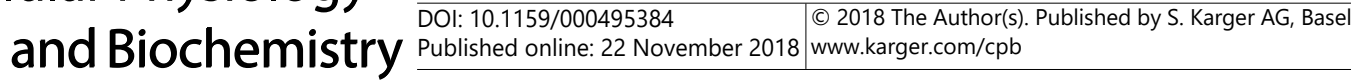 \\ Ma et al.: Microarray Analysis Biomarkers in NAFLD}

may be crucial biomarkers for NAFLD. LncRNA NONMMUT010685 and NONMMUT050689, the regulator of XBP1 gene and RIPK1 gene respectively, played important roles in the development of NAFLD.

\section{Introduction}

Nonalcoholic fatty liver disease (NAFLD), which is now recognized as one of the most common causes of chronic liver disease worldwide, is often identified by serum alanine aminotransferase (ALT), and nonalcoholic hypertransaminasemia, in which viral or other causes of liver disease are excluded [1]. It afflicts more than one third of the population in modern society, paralleling the escalation in global obesity prevalence [2]. A number of pathogenic factors may induce this disease, however, the molecular mechanisms of this disease still remain unknown [3]. Therefore, there is a critical need to better understand the natural molecular characteristics of NAFLD, which could contribute to the better understanding of the pathogenesis of NAFLD and the novel diagnostic markers.

Long non-coding RNAs (lncRNAs) are RNA transcripts longer than 200 nucleotides, without proteins translation capacity [4]. It could either act as transcriptional regulators by silencing through chromatin modification or mediating gene activation [5]. Posttranscriptional regulation of IncRNA includes base pairing of messenger RNA (mRNA) and using decoys of RNA-binding proteins/microRNAs (miRNAs) to suppress splicing [6]. LncRNAs are involved in numerous cellular processes, such as cell growth, differentiation, apoptosis and cancer metastasis [7]. LncRNAs are becoming increasingly focused on pathogenesis of liver diseases and have been shown to have potential diagnostic, prognostic, and therapeutic importance [8, 9]. Our group found Maternally expressed gene 3 (MEG3), a long noncoding RNA, which was expressed in many normal tissues and functioned as a lncRNA tumor suppressor, may play an important role in stellate cell activation and liver fibrosis progression and act as a novel potential therapeutic target for liver fibrosis [10]. However, data on lncRNAs in NAFLD are still scant. Further studies are required to clarify the roles of lncRNA and key genes in NAFLD.

In this study, we performed a genome-wide lncRNA and mRNA microarray analysis to further identify valuable RNAs in NAFLD. Moreover, gene ontology (GO), Kyoto Encyclopedia of Genes and Genomes (KEGG) pathway, construction of IncRNA-mRNA weighted coexpression network, and protein-protein interaction (PPI) network were used to expand the further understanding of NAFLD pathogenesis. As one of the most common stage of NAFLD, NASH rats were further chosen to verify the key protein identified on co-expression network and PPI network data. This work would provide new insights for the therapeutic approaches.

\section{Materials and Methods}

\section{Acquisition of data}

The gene expression data of GSE24031 and GSE57425 were downloaded from the National Center of Biotechnology Information (NCBI) Gene Expression Omnibus (http://www.ncbi.nlm.nih.gov/geo/) database (PMC3531084, PMC2992781). GSE24031 included 4 control mouse liver tissues and 4 model mouse liver tissues, and GSE57425 included 3 control mouse liver tissues and 3 model mouse liver tissues.

Re-annotation of Gene chip probe

A number of lncRNAs represented on the Affymetrix microarrays were identified by the IncRNA classifcation pipeline. Firstly, we obtained the latest version of NetAffx Annotation File Mouse430_2 Annotations, CSV format, Release 36 (24 MB, 4/13/16) from the Affymetrix official website. This annotation file was mapped to the Mouse430_2 probesets ID. Secondly, the IDs beginning with 'NR' were retained, and transcript IDs labeled with 'NP' were deleted from the Refseq database. The online software BioMart 


\section{Cellular Physiology Cell Physiol Biochem 2018;51:871-885

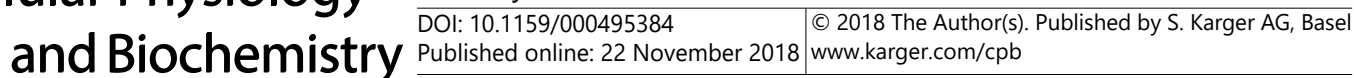 \\ Ma et al.: Microarray Analysis Biomarkers in NAFLD}

was applied to convert Affymetrix microarray IDs to Ensembl IDs together with the corresponding gene type from the Ensembl database. Furthermore, genes annotated as 'IncRNA', 'sense_intronic', 'processed_ transcript', 'antisense', 'sense_overlapping', '3prime_overlapping_ncrna', or 'misc_RNA' were retained from NONCODE database. Finally, 'microRNA', 'snoRNAs', ' pseudogenes' and other small RNAs were removed (PMID: 22709987).

\section{Differential expressions of IncRNAs and mRNAs}

The raw files were normalized using the log scale MAS5 with default settings. After normalization, expression value of each probe for mRNAs or IncRNAs was obtained. Limma (linear models for microarray data) package in R was then chosen to identify differentially expressed IncRNAs and mRNAs between the two groups (PMID: 16646809) [11]. Fold-change> 1.2 and FDR< 0.05 were regarded as the criteria for differential expression. Venny 2.1 was used to identify the up-regulated and down-regulated intersection of genes between GSE24031 and GSE57425 (Oliveros JC, 2007-2015. Venny. An interactive tool for comparing lists with Venn's diagrams. http://bioinfogp.cnb.csic.es/tools/venny/index.html). Hierarchical clustering was performed using the R scripts.

\section{Analysis of GO and KEGG pathways}

Gene ontology (GO) analysis was performed to study large-scale genes. Kyoto Encyclopedia of Genes and Genomes (KEGG) was used to analyze the biological pathways, including the differentially expressed mRNAs. In this study, up and down regulated differentially expressed mRNAs were analyzed by Cytoscapebingo and David software (PMID: 15972284, PMC2615629). $\mathrm{p}<0.05$ was selected as the threshold for significant enrichment terms.

\section{Co-expression network of IncRNA-mRNA}

LncRNA-mRNA-network was established to identify the interactions between mRNA and IncRNA. It was built according to the normalized signal intensity of specific expression of mRNA and IncRNA. For each pair of mRNA-IncRNA, mRNA-mRNA or IncRNA-IncRNA, Pearson correlation was calculated and $\mathrm{P}<0.05$ was chosen as the significant correlation pairs to construct the network.

\section{Construction of PPI network}

The interactions of the proteins were generated by the online String database (Search Tool for the Retrieval of Interacting Genes, http://string-db.org/) and the combined score $>0.4$ was performed as the cut-off criterion (PMC4383874). To display the PPI network, cytoscape (http://cytoscapeweb. cytoscape. org/) was further used (PMC403769).

\section{The establishment of NASH rats}

Male Sprague-Dawley rats $(230 \pm 20 \mathrm{~g})$ were supplied by the Experimental Animal Center, Anhui Medical University. They were housed in plastic cages at a room temperature of $22 \pm 1{ }^{\circ} \mathrm{C}$ under a 12 -h light-dark cycle. The rats were randomly divided into two groups ( $\mathrm{n}=10$ per group): control group and model group with high-fat emulsion (HF group). The method was followed by Yuhong Zou' paper which was published on life science [12]. Our studies were carried out in accordance with the guidelines for humane treatment of animals set by the Association of Laboratory Animal Sciences and the Center for Laboratory Animal Sciences, Anhui Medical University.

\section{Analytical procedures}

The concentration of aspartate aminotransferase (AST), alanine aminotransferase (ALT), total cholesterol (TC), triglyceride (TG) and glucose in the serum were measured by an automatic analyzer. The levels of serum-free fatty acids (FFA), high-density lipoprotein (HDL-C), low-density lipoprotein (LDL-C) were detected with commercial analysis kits obtained from the Jiancheng Institute of Biotechnology (Nanjing, China). 


\section{Cellular Physiology Cell Physiol Biochem 2018;51:871-885

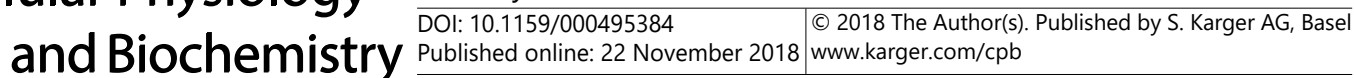 \\ Ma et al.: Microarray Analysis Biomarkers in NAFLD}

Immunoblot analysis

Whole extracts were separated by 10 or $12 \%$ sodium dodecyl sulfate polyacrylamide gel electrophoresis (SDS-PAGE), transferred to a polyvinylidene difluoride membrane, which were incubated with primary antibodies against ATP-citrate lyase (Acly), x-box binding protein 1 (XBP1) and receptor-interacting protein 1 kinase (RIPK1) (Santa Cruz biotechnology, Dallas, TX, USA). The membranes were then washed in TBS/Tween 20 and incubated with secondary antibodies correspondingly. After extensive washing in TBS/Tween 20, protein bands were visualized with ECL chemiluminescent kit (ECL-plus; Thermo Fisher Scientific, Pittsburgh, PA, USA).

\section{Histopathology}

Liver tissues were fixed in $4 \%$ paraformaldehyde for $24 \mathrm{~h}$ immediately following sacrifice, processed for histological examination according to a conventional method, and stained with hematoxylin and eosin (H\&E). Formalinfixed (not embedded in paraffin) and frozen fresh samples were cut in cryostat for intracellular lipid detection by staining with Oil Red 0 .

\section{Isolation of primary hepatocytes}

Primary rat hepatocytes were isolated as described by Hirsova et al. [13]. Briefly, primary hepatocytes were isolated from male Sprague Dawley rats by collagenase perfusion and purified by Percoll (Sigma, St. Louis, MO) gradient centrifugation. The liver was perfused with collagenase and isolated hepatocytes were suspended in DMEM-F12 containing 10\% FBS, $1 \mathrm{nM}$ bovine insulin, $100 \mathrm{U} / \mathrm{ml}$ penicillin and $0.1 \mathrm{mg} /$ $\mathrm{ml}$ streptomycin, $50 \mathrm{nM}$ hydrocortisone, $0.15 \mathrm{mg} / \mathrm{ml}$ methionine. Cells $\left(1.2 \times 10^{6} \mathrm{in} 4 \mathrm{ml}\right)$ were plated in individual 60 -mmdiameter LUX culture dishes (coated with $0.03 \%$ rat tail collagen) and cultured in a $5 \% \mathrm{CO}_{2}$ atmosphere at $37^{\circ} \mathrm{C}$. The viability of the isolated hepatocytes was generally greater than $90 \%$ when judged by trypan blue exclusion. After $3 \mathrm{~h}$, the culture medium was changed to serum-free medium containing 100 $\mathrm{U} / \mathrm{ml}$ penicillin and $0.1 \mathrm{mg} / \mathrm{ml}$ streptomycin.

\section{Statistical analysis}

Quantitative data are expressed as mean \pm S.D. and compared using Student's t-test and Mann-Whitney rank sum test where applicable. $\mathrm{P}<0.05$ was considered statistically significant.

\section{Results}

Differential expression of IncRNAs and $m R N A s$

To compare differentially expressed genes in GSE24031 with GSE57425 by the cut-off criteria, 6 significantly up and 39 down-regulated lncRNAs were screened out. Moreover, 340 significantly up and 281 down-regulated mRNAs were observed (Fig. 1A-D). As shown in Fig. 2 and Fig. 3, the Venn diagram and hierarchical clustering were also performed.

\section{Analysis of GO and KEGG pathways}

In order to identify GO enriched functions for significant differentially expressed mRNAs, bingo was performed. As shown in Fig. 4, top 10 GO was listed. Subsequently, down regulated gene go-net and up regulated gene go-net were depicted in Fig. S1A and Fig. S1B (For all supplemental material see www.karger.com/10.1159/000495384/).

Interestingly, KEGG enrichment pathways of up regulated differentially expressed mRNAs were focus on small molecule metabolic process, cellular process, metabolic process, cellular metabolic process, etc., while down-regulated differentially expressed mRNAs were focus on metabolic process, cellular process, macromolecule metabolic process, cellular metabolic process, etc.. The top 10 pathway results were listed in Table 1 and Fig. 5. 

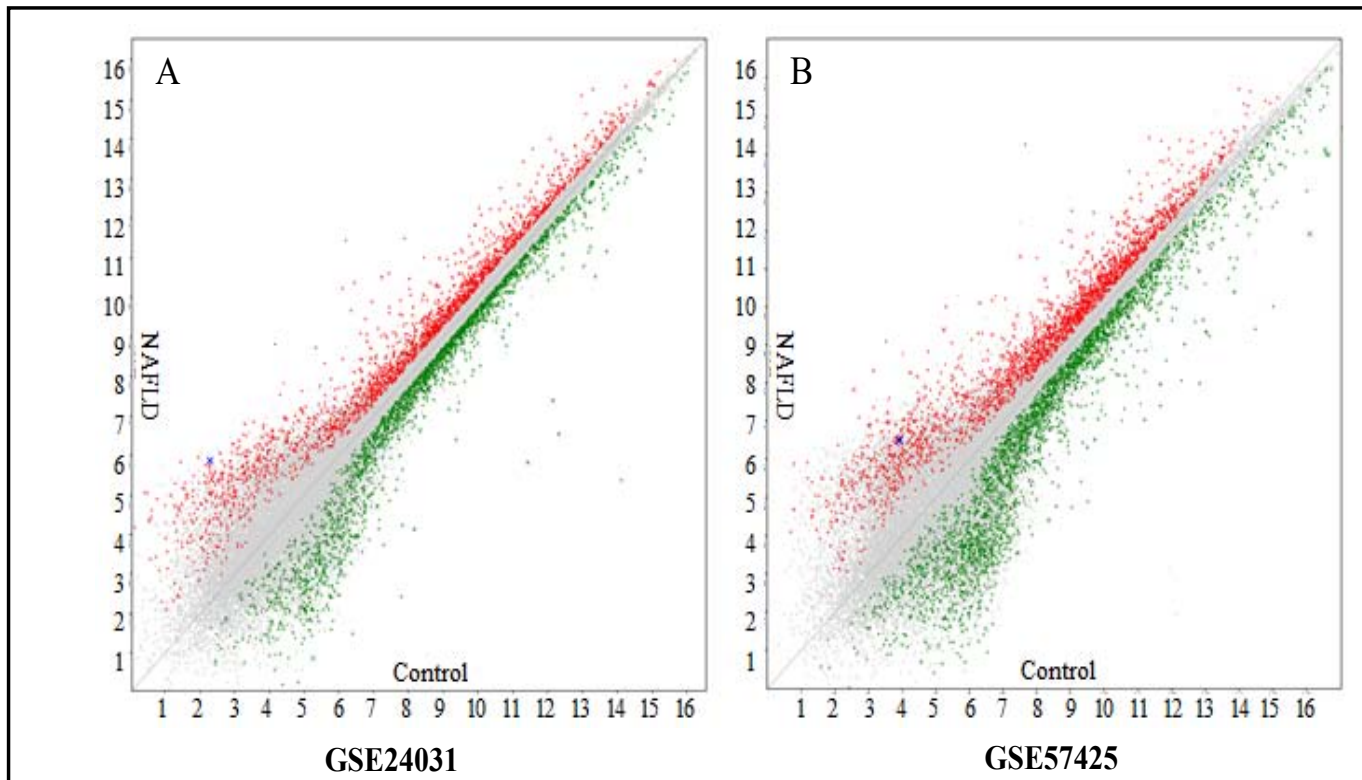

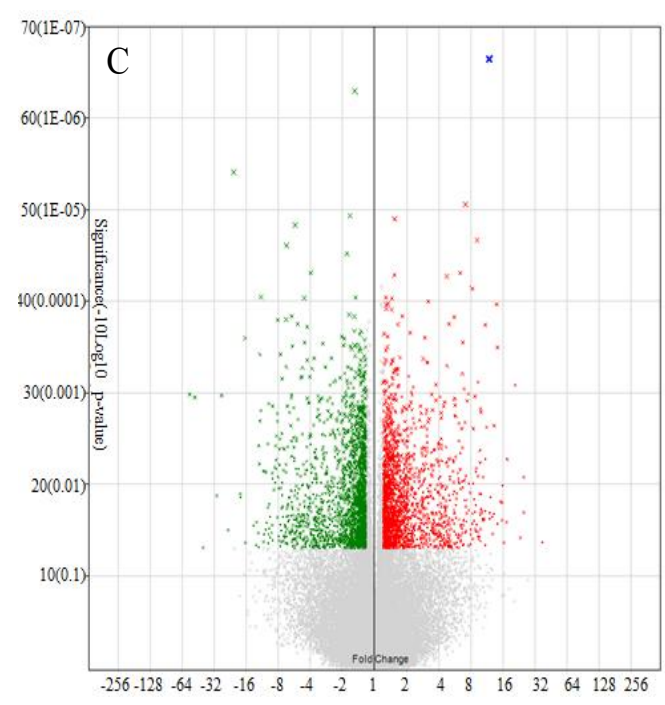

GSE24031

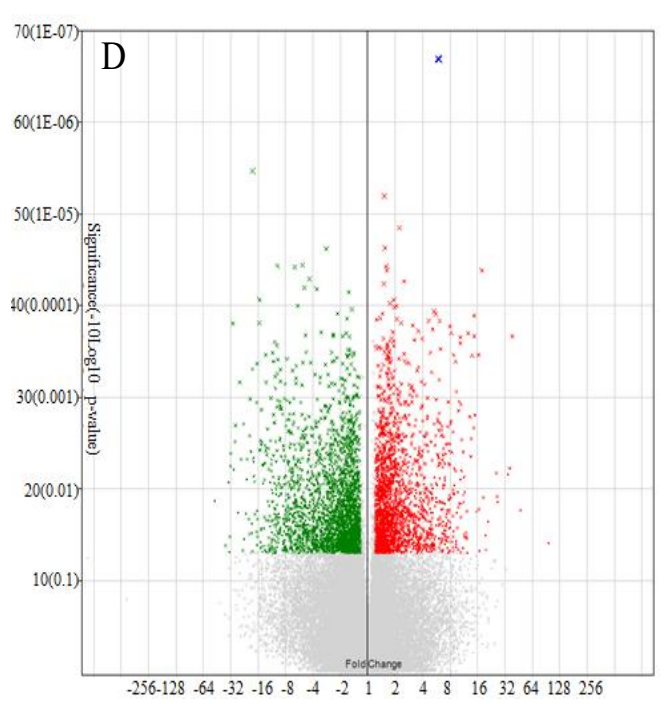

GSE57425

Fig. 1. Scatter-plot and Volcano plots analysis of the microarray data between the control and HF group. Red and green points standed for differential expression of IncRNAs and mRNAs. A. Scatter-plot analysis of the microarray data on the differentially expressed lncRNA and mRNA in GSE24031 B. Scatter-plot analysis of the microarray data on the differentially expressed lncRNA and mRNA in GSE57425 C. Volcano plots analysis of the microarray data on the differentially expressed lncRNA and mRNA in GSE24031. Volcano plots of fold change values of Probesets vs transformed $(\log 10)$ and FDR of case group relative to control group. D. Volcano plots of the microarray data on the differentially expressed lncRNA and mRNA in GSE57425. Volcano plots of fold change values of Probesets vs transformed (log10) and FDR of case group relative to control group. 


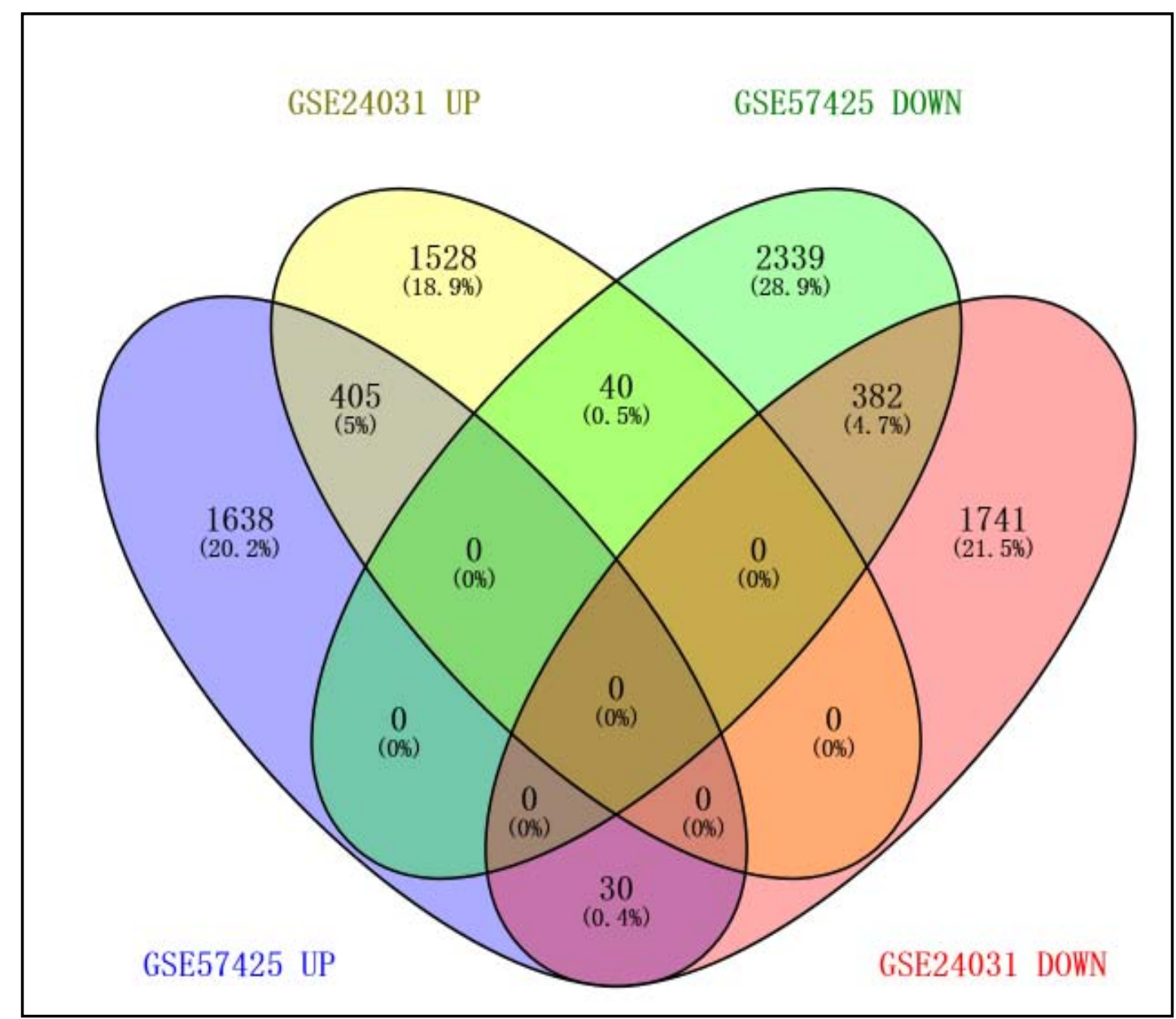

Fig. 2. Venn diagram analysis of up-regulated and down-regulated expressions of lncRNAs and mRNAs in GSE24031 and GSE57425.

\section{Co-expression network of IncRNA-mRNA}

It is demonstrated that genes with the same biological function or pathway may have similar expression patterns, so co-expression network was chosen to provide information about functions of IncRNAs. As shown in Fig. S2A, IncRNA-mRNA co-expression network with statistically differentially expressed lncRNAs and mRNAs was established. Among the network, we chose IncRNA node degree>10 to establish the sub-network (Fig. S2B). Among the network, we found NONMMUT010685 (degree=103), NONMMUT050689 (degree=42), AU021025 (degree=42), AU022434 (degree=31) had higher degrees.

\section{Construction of PPI network}

We examined the PPI network to find significantly up and down-regulated genes by the STRING database. As shown in Fig. S3, several PPI nodes were higher in connectivity degrees, as follows: Acly (ATP citrate lyase, degree=75, fold change (GSE24031)=1.23, fold change (GSE57425)=2.64), Cad (carbamoyl-phosphate synthetase 2, aspartate transcarbamylase, and dihydroorotase, degree $=62$, fold change (GSE24031) $=2.34$, fold change (GSE57425) $=2.54$ ), Eprs (glutamyl-prolyl-tRNA synthetase, degree $=43$, fold change (GSE24031) $=1.29$, fold change (GSE57425)=1.21), Egfr (epidermal growth factor receptor, degree $=42$, fold change (GSE24031) $=3.09$, fold change (GSE57425) $=5.33$ ), Fn1 (fibronectin 1 , degree $=42$, fold change $($ GSE24031 $)=1.23$, fold change $($ GSE57425) $=1.9)$.

\section{KARGER}


$\mathbf{A}$

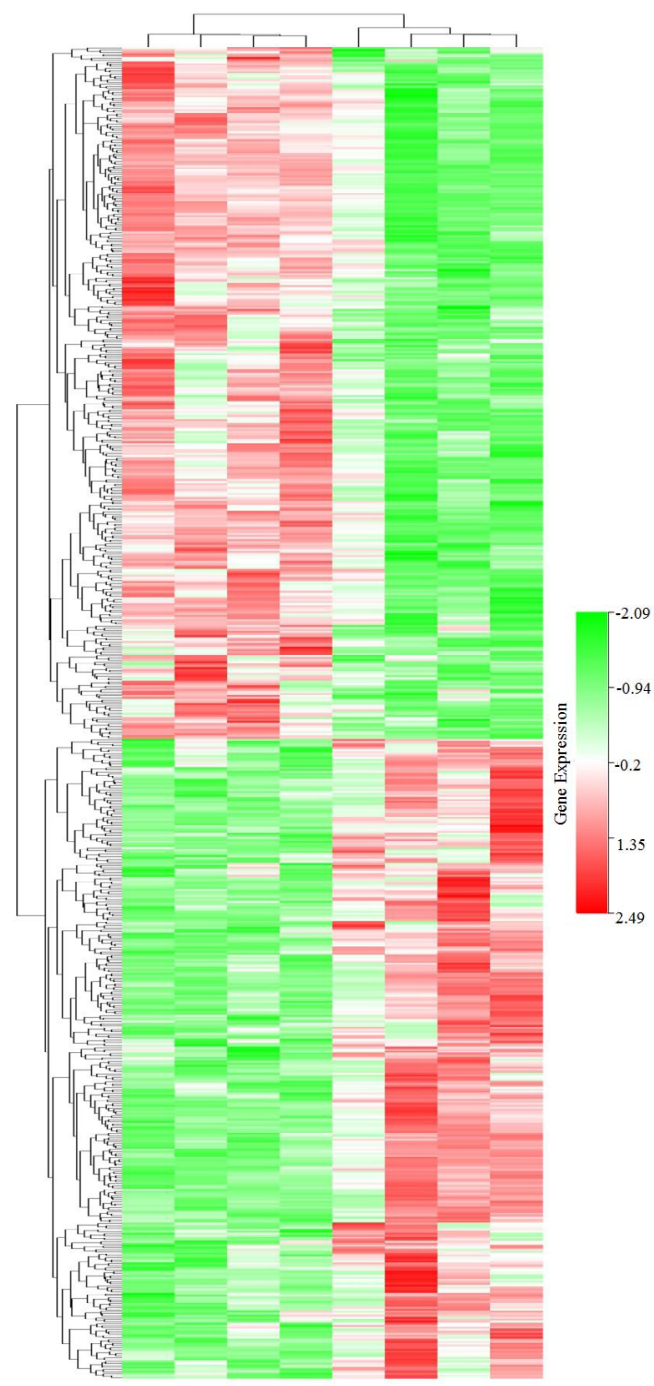

C1 C2 C3 C4 M1M2M3 M4

GSE24031
B

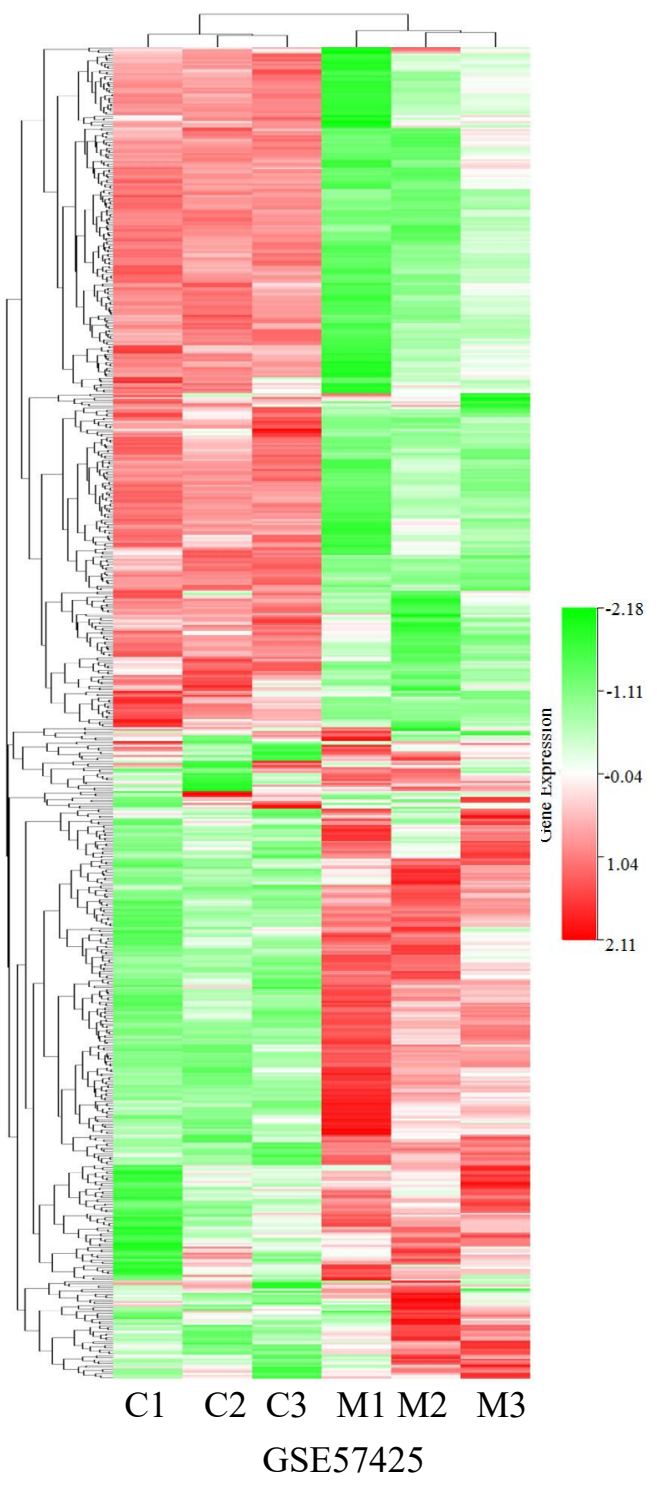

Fig. 3. Heatmap of differentially expressed lncRNAs and mRNAs between the control and HF group. A. Heatmap of differentially expressed IncRNAs and mRNAs in GSE24031.B. Heatmap of differentially expressed IncRNAs and mRNAs in GSE57425. (up-regulated genes were labeled in red, down-regulated genes were labeled in green.)

\section{Serum biochemistry change after high-fat emulsion treatment}

The NASH rats were induced by feeding with high-fat emulsion for 6 weeks, and the general condition of the rats remained satisfactory. Serum levels of AST, ALT, TC, TG, FFA and LDL-C in the HF group were significantly higher than those in control group, whereas the level of HDL-C was lower (Fig. 6. A-C). 


\section{A}

nucleoside phosphate metabolic process nucleotide metabolic process catabolic process lipid metabolic process alcohol metabolic process primary metabolic process cellular metabolic process metabolic process cellular process small molecule metabolic process

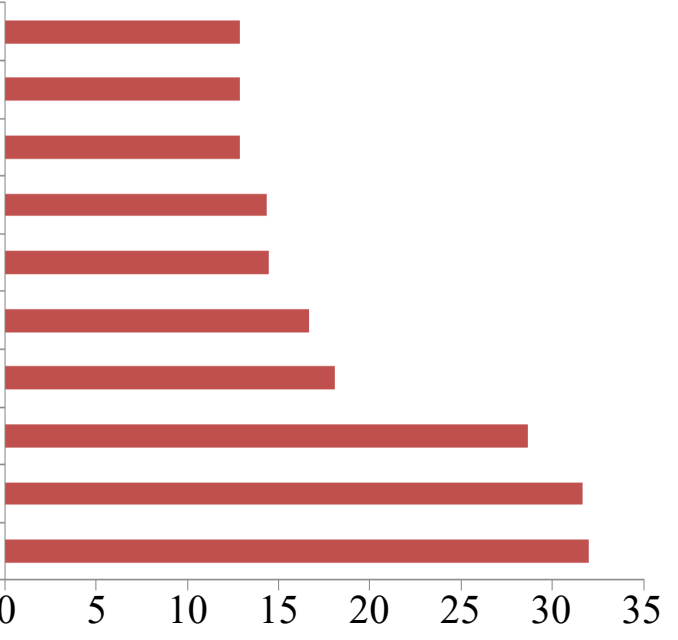
-LOG10(p-value)

\section{B}

nucleobase, nucleoside, nucleotide and nucleic acid metabolic process

regulation of primary metabolic process regulation of cellular metabolic process regulation of metabolic process primary metabolic process cellular macromolecule metabolic process cellular metabolic process macromolecule metabolic process

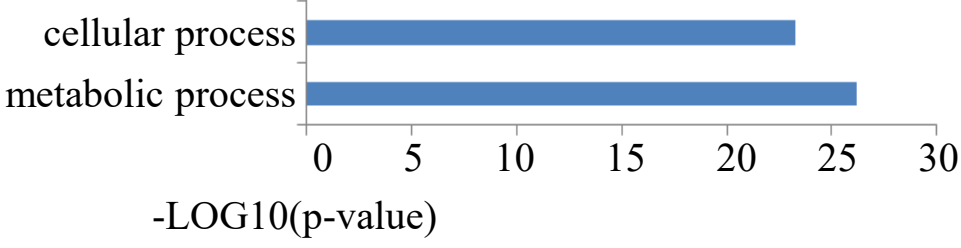

Fig. 4. Biological function by GO analysis of differentially expressed mRNAs. A. The top 10 up-regulated significant changes in GO biological process classification. B. The top 10 down-regulated significant changes in GO biological process classification.

\section{Morphological analysis}

Rats fed with high-fat emulsion for 6 weeks developed a higher degree of steatosis by hematoxylin eosin (H\&E) staining. The liver sections and severe cytoplasmic vacuoles in the hepatocytes were observed in the HF group, while control group showed normal lobular architecture with central veins and radiating hepatic cords (Fig. 6D-E). In line with HE data, the number of lipid droplet was signifcantly increased in liver tissue of NASH rats compared to the control group (Fig. 6F).

\section{KARGER}




\section{Cellular Physiology Cell Physiol Biochem 2018;51:871-885

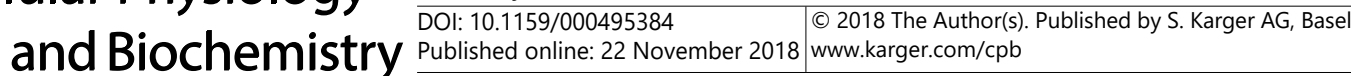

Ma et al.: Microarray Analysis Biomarkers in NAFLD

Table 1. Top 10 up and down-regulated genes related GOs

\begin{tabular}{|c|c|c|c|c|}
\hline $\begin{array}{l}\text { Pathway } \\
\text { Up-regulated }\end{array}$ & Count & $\mathrm{p}$-Value & Fold enrichment & FDR \\
\hline $\begin{array}{l}\text { small molecule metabolic process } \\
\text { cellular process } \\
\text { metabolic process } \\
\text { cellular metabolic process } \\
\text { primary metabolic process } \\
\text { alcohol metabolic process } \\
\text { lipid metabolic process } \\
\text { catabolic process } \\
\text { nucleotide metabolic process } \\
\text { nucleoside phosphate metabolic process } \\
\text { Down-regulated }\end{array}$ & $\begin{array}{c}74 \\
9987 \\
8152 \\
44237 \\
44238 \\
6066 \\
6629 \\
9056 \\
9117 \\
6753\end{array}$ & $\begin{array}{l}1.04 \mathrm{E}-32 \\
2.35 \mathrm{E}-32 \\
2.15 \mathrm{E}-29 \\
8.17 \mathrm{E}-19 \\
2.08 \mathrm{E}-17 \\
3.36 \mathrm{E}-15 \\
4.51 \mathrm{E}-15 \\
1.36 \mathrm{E}-13 \\
1.36 \mathrm{E}-13 \\
1.36 \mathrm{E}-13\end{array}$ & $\begin{array}{l}31.98163 \\
31.62841 \\
28.66706 \\
18.08791 \\
16.68198 \\
14.47413 \\
14.34551 \\
12.86624 \\
12.86614 \\
12.86614\end{array}$ & $\begin{array}{l}2.08 \mathrm{E}-29 \\
2.35 \mathrm{E}-29 \\
1.43 \mathrm{E}-26 \\
4.08 \mathrm{E}-16 \\
8.30 \mathrm{E}-15 \\
1.12 \mathrm{E}-12 \\
1.29 \mathrm{E}-12 \\
2.72 \mathrm{E}-11 \\
2.72 \mathrm{E}-11 \\
2.72 \mathrm{E}-11\end{array}$ \\
\hline $\begin{array}{l}\text { metabolic process } \\
\text { cellular process } \\
\text { macromolecule metabolic process } \\
\text { cellular metabolic process } \\
\text { cellular macromolecule metabolic process } \\
\text { primary metabolic process } \\
\text { regulation of metabolic process } \\
\text { regulation of cellular metabolic process } \\
\text { regulation of primary metabolic process } \\
\text { nucleobase, nucleoside, nucleotide and nucleic acid metabolic process }\end{array}$ & $\begin{array}{c}8152 \\
9987 \\
43170 \\
44237 \\
44260 \\
44238 \\
19222 \\
31323 \\
80090 \\
6139\end{array}$ & $\begin{array}{l}7.00 \mathrm{E}-27 \\
5.80 \mathrm{E}-24 \\
1.36 \mathrm{E}-21 \\
1.40 \mathrm{E}-21 \\
9.68 \mathrm{E}-21 \\
1.01 \mathrm{E}-20 \\
7.50 \mathrm{E}-17 \\
1.64 \mathrm{E}-15 \\
2.22 \mathrm{E}-15 \\
3.12 \mathrm{E}-15\end{array}$ & $\begin{array}{l}26.15485233 \\
23.23681168 \\
20.86690839 \\
20.85244734 \\
20.01415156 \\
19.99705679 \\
16.12521677 \\
14.78544754 \\
14.65417554 \\
14.50569232\end{array}$ & $\begin{array}{l}1.29 \mathrm{E}-23 \\
5.34 \mathrm{E}-21 \\
6.47 \mathrm{E}-19 \\
6.47 \mathrm{E}-19 \\
3.09 \mathrm{E}-18 \\
3.09 \mathrm{E}-18 \\
1.97 \mathrm{E}-14 \\
3.78 \mathrm{E}-13 \\
4.54 \mathrm{E}-13 \\
5.75 \mathrm{E}-13\end{array}$ \\
\hline
\end{tabular}

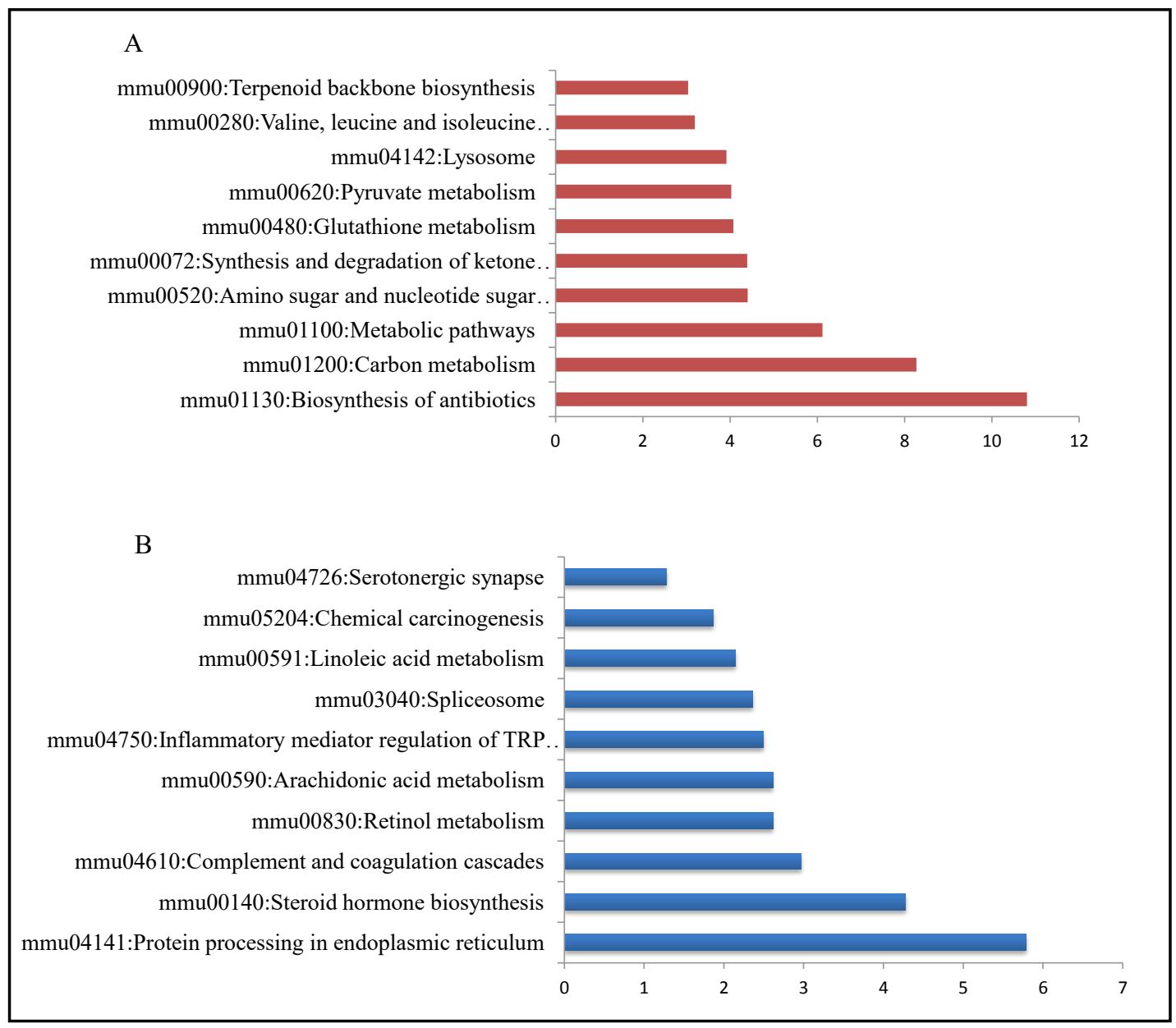

Fig. 5. Pathways characterized from the differentially expressed mRNAs in NAFLD. A. The top 10 upregulated significant changes in pathway. B. The top 10 down-regulated significant changes in pathway. 


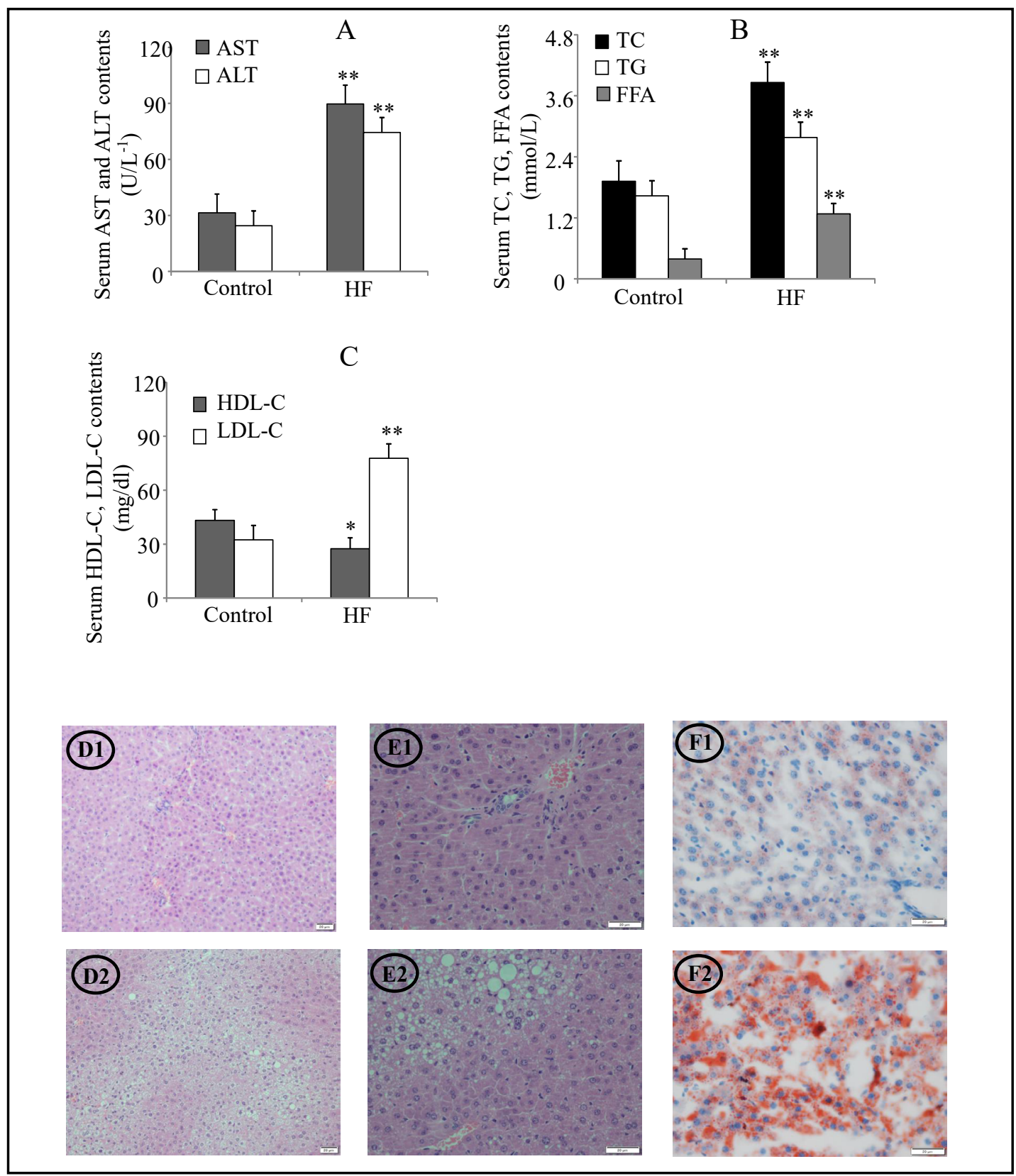

Fig. 6. Serum biochemical indices change and morphological analysis after high-fat emulsion treatment for 6 weeks. A. Serum content of AST and ALT changes after high-fat emulsion treatment for 6 weeks. AST and ALT concentrations were significantly higher in HF group than those in control group. Values are given as means \pm S.D. of 10 rats. ${ }^{* *} \mathrm{P}<0.01$ compared with control group. B. Serum content of TC,TG and FFA changes after high-fat emulsion treatment for 6 weeks. TC,TG and FFA concentrations were significantly higher in HF group than those in control group. Values are given as means \pm S.D. of 10 rats. ${ }^{* *} \mathrm{P}<0.01$ compared with control group. C. Serum content of HDL-C and LDL-C changes after high-fat emulsion treatment for 6 weeks. HDL-C concentrations were significantly lower in HF group than those in control group while LDL-C concentrations were significantly higher. Values are given as means \pm S.D. of 10 rats. ${ }^{*} \mathrm{P}<0.05,{ }^{*} \mathrm{P}<0.01$ compared with control group. D. The liver samples were stained with hematoxylin-eosin (magnification $\times 20$ ). D1. The liver samples from control group; D2. The liver samples from HF group; E. The liver samples were stained with hematoxylin-eosin (magnification $\times 40$ ). E1. The liver samples from control group; E2. The liver samples from HF group; F. The liver samples were stained with Oil red $O$ (magnification $\times 40$ ). F1. The liver samples from control group; F2. The liver samples from HF group. 
Fig. 7. Immunoblot analysis revealed the protein expressions of Acly, XBP1 and RIPK1 in rat liver tissues. A. The protein expression of Acly was significantly increased in the HF group compared with control group. B. The expression of XBP1 was decreased in $\mathrm{HF}$ group. ${ }^{* *} \mathrm{P}<0.01$ compared with control group. C. The expression of RIPK1 was decreased in HF group. ${ }^{* *} \mathrm{P}<0.01$ compared with control group.

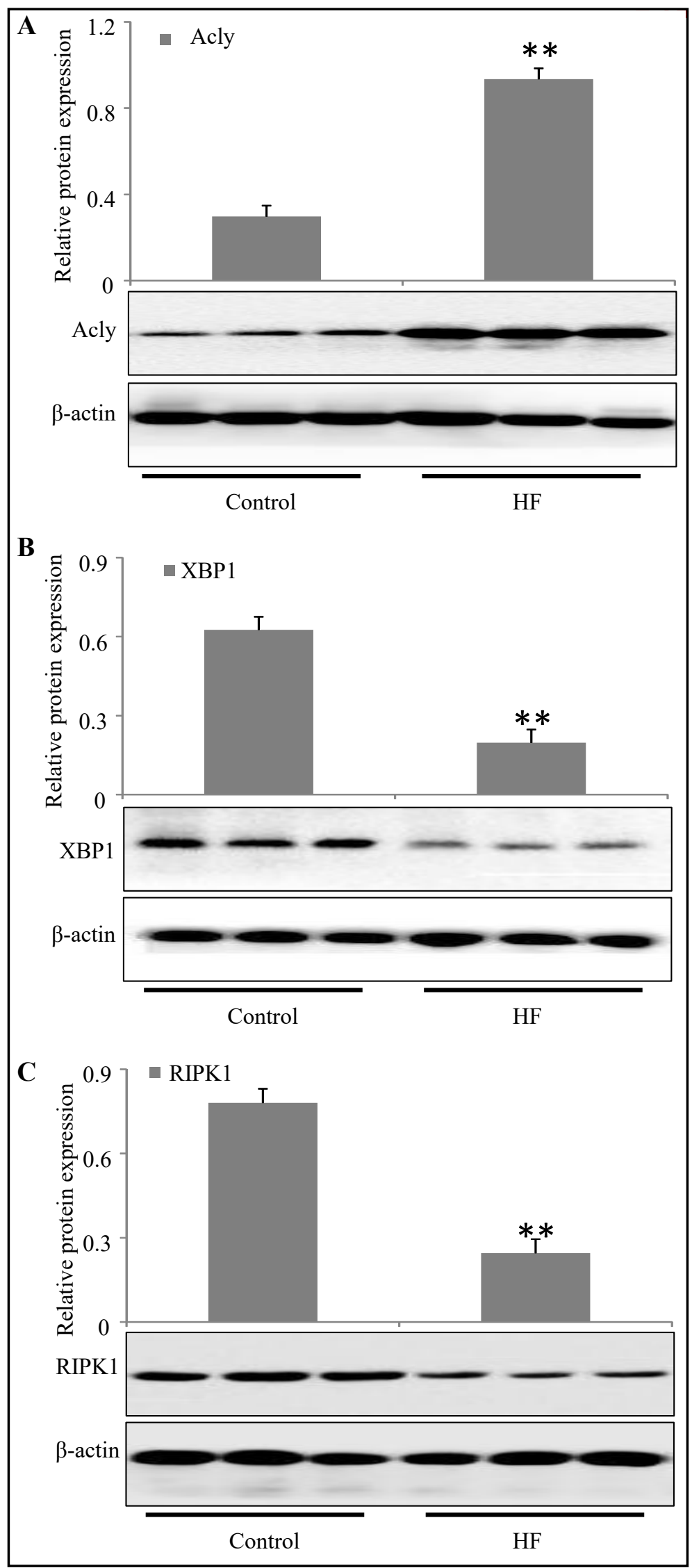




\section{Cellular Physiology Cell Physiol Biochem 2018;51:871-885

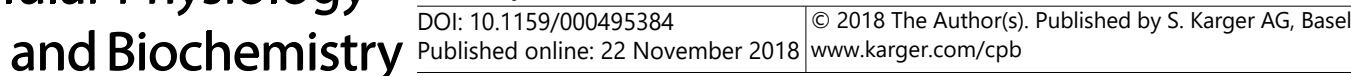

Fig. 8. Immunoblot analysis revealed the protein expression of Acly, XBP1 and RIPK1 in primary hepatocytes of NASH. Wholecell extracts were isolated from primary hepatocytes of control rats and NASH rats, and Western blotting was performed to examine the protein levels of Acly, XBP1 and BIPK1. Data are representative of three independent experiments. $* * \mathrm{P}<0.01$ versus control.

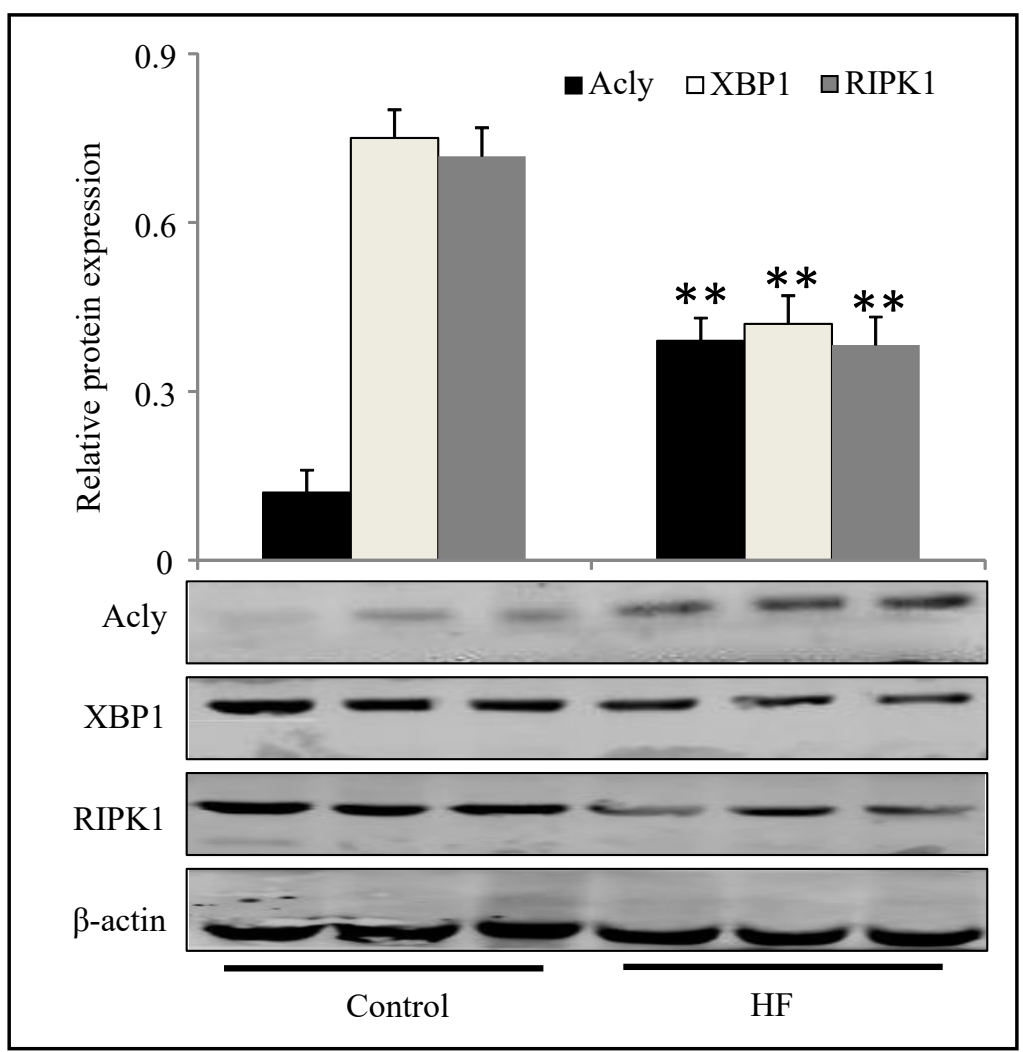

Expressions of Acly, XBP1 and RIPK1 in liver tissues and in primary hepatocytes

As Acly had the highest connectivity degree in PPI network, the expression of Acly was verified in NASH rats. During the 6 weeks of diet treatment, the protein level of Acly was significantly increased in liver tissues and primary hepatocytes in HF group (Fig. 7A, Fig. 8). Furthermore, LncRNA-mRNA co-expression network revealed two key lncRNAs (node degree >10), NONMMUT010685 and NONMMUT050689 in NAFLD samples, whose regulator gene was XBP1 and RIPK1 respectively. It was demonstrated that XBP1 and RIPK1 protein expressions were gradually reduced in liver tissues and primary hepatocytes in HF group compared with control group (Fig. 7B-C, Fig. 8).

\section{Discussion}

The present study identified 340 significantly up and 281 down-regulated mRNAs. In PPI network, up-regulated Acly had the highest connectivity degree. In addition, 6 significantly up-regulated (Ttc39aos1, NONMMUT002658, Clec7a, AV271276, 4931408D14Rik, 1110020A21Rik) and 39 down-regulated IncRNAs were detected. LncRNA-mRNA coexpression network revealed a total of 16 key lncRNAs (node degree $>10$ ) in NAFLD samples, such as NONMMUT010685, NONMMUT050689, AU021025, AU022434, et al..

NAFLD is now recognized as the most common type of liver disease and might lead to important public health problems. This disease, with an unclear natural history, can be severe and is characterized by a wide spectrum of pathological lesions. It ranges from uncomplicated hepatic steatosis to hepatocellular carcinoma. NASH is a chronic liver disease characterized by excessive triglyceride accumulation in the liver accompanied by inflammation, cell stress and apoptosis [14]. It is the tipping point to the life-threatening stages of NAFLD. Thus we chose NASH model, to some degree, to reflect the natural multi-factorial etiological setting of the most common form of NAFLD. 


\section{Cellular Physiology Cell Physiol Biochem 2018;51:871-885

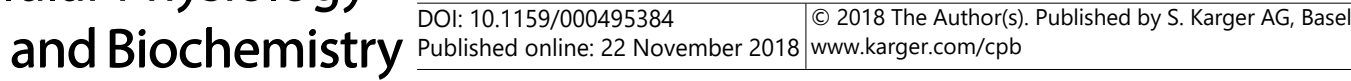 \\ Ma et al.: Microarray Analysis Biomarkers in NAFLD}

As NAFLD is the hepatic manifestation of metabolic syndrome and inflammation $[15,16]$, the up-regulation of Acly and LncRNA Ttc39aos1 gave rise to our attention. Acly catalyzes conversion of citrate into acetyl-CoA, the building block for the endogenous biosynthesis of fatty acids [17]. In mice, Acly is highly expressed in lipogenic tissues such as liver, kidney, pancreatic beta cells, and brain [18]. Acly activity has been related to metabolic disorders such as dyslipidemia, hepatic steatosis, and impaired glucose tolerance $[19,20]$. It was reported that leptin deficient db mice significantly increased hepatic Acly expression, and liver-specific Acly knockdown ( $>95 \%$ ) attenuated hepatic steatosis, inhibited hepatic lipogenesis, and down-regulated liver gluconeogenic gene expression [21]. In another research, high-fat fed C57BL/6 mice whose liver-specific Acly knockdown (>88\%) decreased plasma triglyceride, and inhibited hepatic secretion of very low density lipoprotein (VLDL) triglyceride and apoB48 [20]. In Ahrens' research, it was suggested that Acly was involved in the course of NASH by DNA methylation analysis in NASH patients [17]. In this paper, it was demonstrated that the level of Acly was up-regulated in NASH rats in our study. These studies, accompanied with the important role of Acly in fatty acid and cholesterol synthesis in lipogenic tissues, indicated modulating its activity may play a potential role in treatment of metabolic syndrome.

LncRNA Ttc39aos1, a 2.5-kb spliced and polyadenylated IncRNA, was originally discovered due to its induction during mouse erythropoiesis [22]. As an important transcriptional brake, it curbs inflammatory gene expression in macrophages and in mice [6]. It was found that Ttc39aos1 was down-regulated in macrophages exposed to inflammatory stimuli. Precise regulation of Ttc39aos1 expression in macrophages was essential to control inflammatory responses by suppressing the transcription of key immune genes [23].

In co-expression network, LncRNA NONMMUT010685 was found to regulate XBP1 gene. XBP1, a key regulator of the unfolded protein response, was required for the unrelated function of normal fatty acid synthesis in the liver. As a regulator of lipogenesis, Lee's group reported XBP1 had important implications for human dyslipidemias [24]. It was also reported, XBP1 was required for development and maintenance of secretory cells and linked to JNK activation. It linked ER stress to intestinal inflammation and conferred genetic risk for human inflammatory bowel disease [25]. In Fuchs' group research, it was reported that continuous excessive availability of hepatic saturated fatty acids (SFAs) were likely to generate lipid intermediates and shift normal triacylglyceride formation to induction of ER stress. Failure to efficiently degrade proteins in response to activating X-box protein 1 (XBP1) may identify NASH patients at particular risk to progress to cirrhosis [26]. In our study, we also found IncRNA NONMMUT050689 could regulate RIPK1 gene in co-expression network of lncRNA-mRNA. RIPK1 is implicated in inflammatory and cell death pathway and its kinase activity is reported to drive RIPK3-mediated necroptosis. Dannappel's team found it could inhibit RIPK3-mediated necroptosis in vivo and identified necroptosis as a more potent trigger of inflammation [27]. Furthermore, Farooq's group investigated the role of RIPK1 in NASH. It was found RIPK1 in hepatocyte limited the progression of liver fibrosis during NASH[28]. Our research demonstrated that the expressions of XBP1 and RIPK1 were downregulated in NASH, suggesting XBP1 and RIPK1 participation in the pathogenesis of NASH.

Although the underlying mechanisms of these key lncRNAs in NAFLD have not been fully discussed, our results indicated their contributions to NAFLD development to some extent.

\section{Conclusion}

In conclusion, NAFLD was a complicated process with many lncRNAs, mRNAs and pathways involved. Acly may be pivotal biomarkers for NAFLD. LncRNA NONMMUT010685 and NONMMUT050689, regulator of XBP1 gene and RIPK1 gene respectively, play important roles in the development of NAFLD. This study revealed crucial information on the molecular mechanisms of NAFLD and would provide insights into the important roles of lncRNAs and mRNAs in NAFLD. 


\section{Cellular Physiology Cell Physiol Biochem 2018;51:871-885 \begin{tabular}{ll|l} 
and Biochemistry Published online: 22 November 2018 & $\begin{array}{l}\text { (c) } 2018 \text { The Author(s). Published by S. Karger AG, Basel } \\
\text { www.karger.com/cpb }\end{array}$ \\
\hline
\end{tabular} \\ Ma et al.: Microarray Analysis Biomarkers in NAFLD}

\section{Acknowledgements}

This project was supported by key grants from the National Natural Science Foundation of China (No. 81473268), Educational Commission of Anhui Province (No. KJ2016A364, KJ2016A365) and key projects of outstanding youth foundation in Colleges of Anhui Province of China (No. gxyqZD2016048). We are grateful to Mr. Qiang Fan (Ao Ji Bio-tech Co., Ltd., Shanghai, China) for helps in data analysis.

\section{Disclosure Statement}

The authors declare no conflicts of interest.

\section{References}

1 Tilg H, Moschen AR, Roden M: NAFLD and diabetes mellitus. Nat Rev Gastroenterol Hepatol 2017;14:32-42.

-2 Rinella M, Charlton M: The globalization of nonalcoholic fatty liver disease: Prevalence and impact on world health. Hepatology 2016;64:19-22.

-3 LaGory EL, Giaccia AJ: Long-range hypoxia signaling in NAFLD. Nat Med 2017;23:1251-1252.

4 Mercer TR, Dinger ME, Mattick JS: Long non-coding RNAs: insights into functions. Nat Rev Genet 2009;10:155-159.

5 Fatica A, Bozzoni I: Long non-coding RNAs: new players in cell differentiation and development. Nat Rev Genet 2014;15:7-21.

6 Kopp F, Mendell JT: Functional Classification and Experimental Dissection of Long Noncoding RNAs. Cell 2018;172:393-407.

7 Shi X, Sun M, Liu H, Yao Y, Song Y: Long non-coding RNAs: a new frontier in the study of human diseases. Cancer Lett 2013;339:159-166.

$>8$ Huarte M: The emerging role of IncRNAs in cancer. Nat Med 2015;21:1253-1261.

-9 Li P, Ruan X, Yang L, Kiesewetter K, Zhao Y, Luo H, Chen Y, Gucek M, Zhu J, Cao H: A liver-enriched long noncoding RNA, IncLSTR, regulates systemic lipid metabolism in mice. Cell Metab 2015;21:455-467.

10 He Y, Wu YT, Huang C, Meng XM, Ma TT, Wu BM, Xu FY, Zhang L, Lv XW, Li J: Inhibitory effects of long noncoding RNA MEG3 on hepatic stellate cells activation and liver fibrogenesis. Biochim Biophys Acta 2014;1842:2204-2215.

11 Chen Y, Ni H, Zhao Y, Chen K, Li M, Li C, Zhu X, Fu Q: Potential Role of IncRNAs in Contributing to Pathogenesis of Intervertebral Disc Degeneration Based on Microarray Data. Med Sci Monit 2015;21:34493458.

12 Zou Y, Li J, Lu C, Wang J, Ge J, Huang Y, Zhang L, Wang Y: High-fat emulsion-induced rat model of nonalcoholic steatohepatitis. Life Sci 2006;79:1100-1107.

>13 Hirsova P, Ibrahim SH, Krishnan A, Verma VK, Bronk SF, Werneburg NW, Charlton MR, Shah VH, Malhi H, Gores GJ: Lipid-Induced Signaling Causes Release of Inflammatory Extracellular Vesicles From Hepatocytes. Gastroenterology 2016;150:956-967.

14 Greenhill C: NASH: Understanding how steatosis progresses to NASH. Nat Rev Endocrinol 2017;13:5.

15 Lonardo A, Ballestri S, Marchesini G, Angulo P, Loria P: Nonalcoholic fatty liver disease: a precursor of the metabolic syndrome. Dig Liver Dis 2015;47:181-190.

16 Henao-Mejia J, Elinav E, Jin C, Hao L, Mehal WZ, Strowig T, Thaiss CA, Kau AL, Eisenbarth SC, Jurczak MJ, Camporez JP, Shulman GI, Gordon JI, Hoffman HM, Flavell RA: Inflammasome-mediated dysbiosis regulates progression of NAFLD and obesity. Nature 2012;482:179-185.

17 Ahrens M, Ammerpohl O, von Schonfels W, Kolarova J, Bens S, Itzel T, Teufel A, Herrmann A, Brosch M, Hinrichsen H, Erhart W, Egberts J, Sipos B, Schreiber S, Hasler R, Stickel F, Becker T, Krawczak M, Rocken C, Siebert R, Schafmayer C, Hampe J: DNA methylation analysis in nonalcoholic fatty liver disease suggests distinct disease-specific and remodeling signatures after bariatric surgery. Cell Metab 2013;18:296-302.

18 Burke AC, Huff MW: ATP-citrate lyase: genetics, molecular biology and therapeutic target for dyslipidemia. Curr Opin Lipidol 2017;28:193-200. 


\section{Cellular Physiology Cell Physiol Biochem 2018;51:871-885

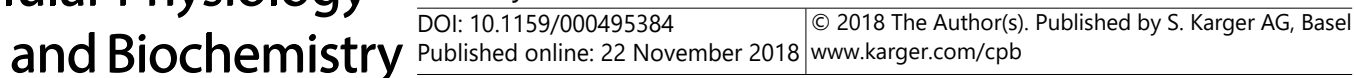 \\ Ma et al.: Microarray Analysis Biomarkers in NAFLD}

19 MacDonald MJ, Longacre MJ, Langberg EC, Tibell A, Kendrick MA, Fukao T, Ostenson CG: Decreased levels of metabolic enzymes in pancreatic islets of patients with type 2 diabetes. Diabetologia 2009;52:1087-1091.

20 Wang Q, Li S, Jiang L, Zhou Y, Li Z, Shao M, Li W, Liu Y: Deficiency in hepatic ATP-citrate lyase affects VLDLtriglyceride mobilization and liver fatty acid composition in mice. J Lipid Res 2010;51:2516-2526.

21 Wang Q, Jiang L, Wang J, Li S, Yu Y, You J, Zeng R, Gao X, Rui L, Li W, Liu Y: Abrogation of hepatic ATPcitrate lyase protects against fatty liver and ameliorates hyperglycemia in leptin receptor-deficient mice. Hepatology 2009;49:1166-1175.

-22 Hu W, Yuan B, Flygare J, Lodish HF: Long noncoding RNA-mediated anti-apoptotic activity in murine erythroid terminal differentiation. Genes Dev 2011;25:2573-2578.

-23 Atianand MK, Hu W, Satpathy AT, Shen Y, Ricci EP, Alvarez-Dominguez JR, Bhatta A, Schattgen SA, McGowan JD, Blin J, Braun JE, Gandhi P, Moore MJ, Chang HY, Lodish HF, Caffrey DR, Fitzgerald KA: A Long Noncoding RNA lincRNA-EPS Acts as a Transcriptional Brake to Restrain Inflammation. Cell 2016;165:1672-1685.

24 Lee AH, Scapa EF, Cohen DE, Glimcher LH: Regulation of hepatic lipogenesis by the transcription factor XBP1. Science 2008;320:1492-1496.

25 Kaser A, Lee AH, Franke A, Glickman JN, Zeissig S, Tilg H, Nieuwenhuis EE, Higgins DE, Schreiber S, Glimcher LH, Blumberg RS: XBP1 links ER stress to intestinal inflammation and confers genetic risk for human inflammatory bowel disease. Cell 2008;134:743-756.

26 Fuchs M, Sanyal AJ: Lipotoxicity in NASH. J Hepatol 2012;56:291-293.

-27 Dannappel M, Vlantis K, Kumari S, Polykratis A, Kim C, Wachsmuth L, Eftychi C, Lin J, Corona T, Hermance N, Zelic M, Kirsch P, Basic M, Bleich A, Kelliher M, Pasparakis M: RIPK1 maintains epithelial homeostasis by inhibiting apoptosis and necroptosis. Nature 2014;513:90-94.

-28 Farooq M, Piquet-Pellorce C, DionM. S, EugenioK. S, Santamaria A, FilliolM T, Dimanche-Boitrel M, Samson J, Seyec L: RIPK1 depletion exacerbates progression of liver fibrosis in high fat diet induced non-alcoholic steatohepatitis (NASH) in mice. Journal of Hepatology 2018;68:Supplement 1 S345. 